\title{
Resistência do solo à penetração em sistemas de preparo e velocidades de deslocamento do trator
}

\author{
Hideo de Jesus Nagahama', Jorge Wilson Cortez², Wisy Alves Pimenta', \\ Antonio Pereira Patrocinio Filho', Elder Barboza de Souza' \\ 'Universidade Federal do Vale do São Francisco, Petrolina, PE, Brasil \\ 2Universidade Federal da Grande Dourados, Dourados, MS, Brasil \\ Autor correspondente, e-mail: jorgecortez@ufgd.edu.br
}

\begin{abstract}
Resumo
O uso de equipamentos de preparo do solo continuamente, sem variação da profundidade de trabalho, pode ocasionar problemas de compactação do solo. Objetivou-se avaliar a variabilidade espacial da resistência do solo à penetração (RP) no perfil de um Argissolo Amarelo em sistemas de preparo do solo e velocidades de deslocamento do conjunto trator-equipamento. $O$ trabalho foi conduzido no Campus de Ciências Agrárias - UNIVASF, Petrolina-PE, em delineamento em blocos ao acaso com parcelas subdivididas e quatro repetições. Os tratamentos foram constituídos por cinco sistemas de preparo do solo: sem preparo primário, grade tandem mais arado de aiveca, grade off-set de discos de 0,56 m, grade off-set de discos de 0,61 m e grade tandem mais escarificador, enquanto que nas subparcelas aplicou-se as velocidades de deslocamento do conjunto tratorequipamento: $2,45 \mathrm{~km} \mathrm{~h}^{-1}$ (L1), 3,88 $\mathrm{km} \mathrm{h}^{-1}$ (L2), 5,72 km h-1 (L3) e 6,50 km h'-1 (H1). Para a coleta de dados de RP utilizou-se um penetrômetro eletrônico e coletaram-se amostras de solo para determinação da umidade no perfil amostral. A análise dos dados foi por meio da geoestatística e dos mapas de isolinhas. Pode-se verificar a área de atuação dos órgãos ativos dos equipamentos, o zoneamento de áreas compactadas (RP) e os níveis de RP no perfil estudado. O sistema grade tandem mais arado de aivecas obteve a menor RP.
\end{abstract}

Palavras-chave: mecanização agrícola, variabilidade espacial, Argissolo Amarelo, compactação do solo

\section{Soil resistance to penetration in tillage systems and tractor speed}

\begin{abstract}
The continuous use of tillage equipment without variation of the work depth can lead to soil compression problems. This study aimed to evaluate the spatial variability of soil resistance to penetration (RP) in a yellow ultisol profile in different tillage systems and tractor-equipment speeds. The experiment was carried out at the Agricultural Sciences Campus - UNIVASF, Petrolina- PE, Brazil, in randomized blocks with split plot with four repetitions. The treatments consisted of five tillage systems applied to the plots: without the first soil preparation, tandem grid and moldboard plow, $0.56 \mathrm{~m}$ offset grid discs, $0.61 \mathrm{~m}$ off-set grid disc plus tandem grid and chisel plow. For the subplots, the speeds of the tractor-equipment used were: $2.45 \mathrm{~km} \mathrm{~h}^{-1}$ (L1), $3.88 \mathrm{~km} \mathrm{~h}^{-1}$ (L2), $5.72 \mathrm{~km} \mathrm{~h}^{-1}$ (L3) and $6.50 \mathrm{~km} \mathrm{~h}^{-1}$ (H1). For the soil data collection and the RP determination, an electronic penetrometer was used, determining the sample profile moisture. Data analysis was carried out based on geostatistics and isoline maps. It can be verified the operationarea of the active organs of the equipment, the zoning of compacted areas (RP) and the RP levels of the studied profile. The lower RP was obtained when the tandem grid plus the chisel plow was used.
\end{abstract}

Key words: agricultural mechanization, spatial variability, yellow ultisoil, soil compression 


\section{Introdução}

A compactação do solo é uma alteração estrutural que promove reorganização das partículas e de seus agregados podendo limitar a adsorção, as trocas gasosas, absorção de nutrientes, infiltração e redistribuição de água, atraso na emergência das plântulas e comprometimento do desenvolvimento do sistema radicular e da parte aérea, resultando em decréscimos na produtividade das culturas (Stone et al., 2002; Modolo et al., 2008).

A resistência do solo à penetração (RP) é um dos atributos físicos que expressa o grau de compactação, e por consequência a facilidade ou não com que as raízes penetram no solo (Mion et al., 2012), uma vez que, a RP se relaciona com outros atributos indicadores do grau de compactação (Stefanoski et al., 2013).

A determinação da RP como indicador da compactação do solo pode ser por meio de penetrômetros (Araújo et al., 2013). Dentre os penetrômetros mais utilizados, está o de impacto por registrar a RP por unidade de profundidade, e permitir a caracterização de solos compactados pelo uso e manejo (Stolf, 1991), permitindo identificar no perfil do solo, barreiras impeditivas ao crescimento radicular das plantas (Magalhães et al. 2009).

O estudo da variabilidade espacial dos atributos físicos do solo utilizando a geoestatística, permite por meio desta análise detectar a variabilidade e distribuição espacial dos atributos estudados e, portanto, constitui importante ferramenta na análise e descrição detalhada da variabilidade dos atributos do solo (Alves et al., 2014). A determinação da variabilidade espacial dos atributos físicos do solo permite o melhor controle dos fatores de produção das culturas e também, do monitoramento ambiental (Oliveira et al., 2013).

Dessa forma, objetivou-se avaliar a variabilidade espacial da resistência do solo à penetração no perfil de um Argissolo Amarelo em sistemas de preparo do solo e velocidades de deslocamento do conjunto trator-equipamento.

\section{Material e Métodos}

O trabalho foi conduzido no Campus de Ciências Agrárias da Universidade Federal do Vale do São Francisco (UNIVASF) em Petrolina - PE, que se localiza na latitude de 09²3' Sul, longitude de 40 $30^{\prime}$ Oeste e altitude de $376 \mathrm{~m}$. Segundo a classificação de Köppen, o clima da região apresenta-se como tropical semiárido, tipo BshW, caracterizado pela escassez e irregularidade das precipitações com chuvas no verão e forte evaporação em consequência das altas temperaturas.

O solo foi classificado como Argissolo Amarelo distrófico típico por Amaral et al. (2006), utilizando o Sistema Brasileiro de Classificação de Solos (Embrapa, 2013). Na Tabela 1 podese verificar a composição granulómetrica do perfil do solo mobilizado e a umidade do solo no momento da coleta de dados da RP. O solo na capacidade de campo apresenta $0,12 \mathrm{~g} \mathrm{~g}^{-1} \mathrm{de}$ umidade.

Tabela 1. Composição granulométrica do Argissolo Amarelo e umidade do solo durante a coleta de RP no perfil da área experimental

\begin{tabular}{ccccc}
\hline \multirow{2}{*}{ Camadas } & \multicolumn{3}{c}{ Granulometria } & \multirow{2}{*}{ Umidade } \\
\cline { 2 - 4 } & Argila & Areia & Silte & \\
\hline (m) & & $\left(\mathrm{kg} \mathrm{kg}^{-1}\right)$ & & $\left(\mathrm{g} \mathrm{g}^{-1}\right)$ \\
$0-0,10$ & 0,090 & 0,878 & 0,032 & 0,08 \\
$0,10-0,20$ & 0,100 & 0,883 & 0,017 & 0,06 \\
$0,20-0,30$ & 0,080 & 0,852 & 0,068 & 0,07 \\
$0,30-0,40$ & 0,140 & 0,807 & 0,053 & 0,07 \\
$0,40-0,50$ & 0,180 & 0,742 & 0,078 & 0,07 \\
\hline Fonte: Adaptado de Cortez et al. (2011) & & &
\end{tabular}

Na área experimental cultivou-se sorgo forrageiro que foi submetido a três cortes para obtenção de forragem até o ano de 2009. Após este periodo, a mesma, continuou em pousio até a instalação do experimento em 2011. Existe na área experimental, sistema linear de irrigação, que propiciou umidade do solo no momento do preparo e na coleta de dados. 
Como fonte de potência para tracionar os equipamentos agrícolas (Tabela 2), foi utilizado o trator da marca Valtra, 785 TDA, com $55,2 \mathrm{~kW}$ (75 cv), com pneus dianteiros 12.4- $24 \mathrm{R} 1$ e traseiros 18.4 - $30 \mathrm{R} 1$, e rotação de trabalho no motor a $2.040 \mathrm{rpm}$.

Para a montagem do experimento foi utilizado o delineamento em blocos ao acaso com parcelas subdivididas e quatro repetições. Os cinco tratamentos de preparo do solo aplicados nas parcelas principais foram: grade tandem mais arado de aivecas - GT + AA, grade leve off-set com discos de $0,56 \mathrm{~m}$ (22 polegadas) - GL22, grade leve off-set com discos de $0,61 \mathrm{~m}$ (24 polegadas) - GL24 e grade tandem mais escarificador de três hastes - GT + ESC, e sem preparo primário (apenas com grade em tandem antes da semeadura). Nas subparcelas aplicaram-se quatro velocidades de deslocamento durante a mobilização do solo, com cada equipamento, sendo: 2,$45 ; 3,88$; 5,72 e $6,50 \mathrm{~km} \mathrm{~h}^{-1}$.

Tabela 2. Equipamentos utilizados para o preparo do solo

\begin{tabular}{|c|c|c|c|c|}
\hline \multirow{2}{*}{ Implementos } & \multirow{2}{*}{ Órgãos ativos } & Massa & Profundidade & Largura \\
\hline & & $(\mathrm{kg})$ & (m) & (m) \\
\hline Arado de aivecas & Duas aivecas recortadas & 570 & 0,42 & 0,90 \\
\hline $\begin{array}{l}\text { Grade leve em } \\
\text { tandem }\end{array}$ & $\begin{array}{l}\text { Sete discos em cada uma das quatro seções, } \\
\text { discos recortados na dianteira lisos na traseira, } \\
\text { diâmetro de } 0,51 \mathrm{~m} \text {. }\end{array}$ & 528 & 0,10 & 2,62 \\
\hline Grade leve off-set & $\begin{array}{l}\text { Oito discos em cada uma das duas seções, } \\
\text { discos recortados com } 0,56 \text { m de diâmetro. }\end{array}$ & 1.000 & 0,15 & 1,73 \\
\hline Grade leve off-set & $\begin{array}{l}\text { Sete discos em cada uma das duas seções, } \\
\text { discos recortados com diâmetro de } 0,61 \mathrm{~m} \text {. }\end{array}$ & 1.094 & 0,18 & 1,50 \\
\hline Escarificador & $\begin{array}{l}\text { Três hastes espaçadas com ponteira estreita } \\
\text { de } 0,05 \mathrm{~m}\end{array}$ & 295 & 0,35 & 1,20 \\
\hline
\end{tabular}

Para a coleta da RP de modo espacial no perfil do solo foi utilizado o Penetrômetro eletrônico denominado de PenetroLOG® PLG1020 - Medidor Eletrônico de Compactação do Solo da empresa Falker Automação Agrícola (Falker, 2010). O medidor segue as recomendações da norma internacional ASAE S.313.3 (ASAE, 2004). As avaliações foram em função da largura de trabalho de cada equipamento ao longo do perfil da área de preparo, sendo a coleta transversal ao deslocamento do conjunto trator-equipamento a cada 0,15 $\mathrm{m}$ e nas profundidades de 0-0,10; 0,10-0,20; 0,0-0,30; 0,30-0,40; e 0,40-0,50 m.

Os dados de RP coletados foram submetidos a análise geoestatística, conforme recomendado por Vieira (2000). Foram construídos semivariogramas, em virtude de pressuposições de estacionaridade intrínseca e do cálculo da semivariância e dos ajustes dos modelos teóricos. O ajuste do semivariograma foi observado pelo maior valor do coeficiente de determinação $\left(R^{2}\right)$, pelo menor valor da soma de quadrados dos desvios (SQR) e pelo maior valor da dependência espacial (ADE). Esse ajuste foi dito como "a sentimento", o qual se escolheu o modelo e respectivos parâmetros, e esse é sobreposto aos pontos estimados, verificando-se a melhor adequação. O modelo escolhido foi submetido à análise comparativa por meio da validação cruzada. Para obtenção dos mapas de isolinhas (bidimensionais) foi utilizado um interpolador, a krigagem ordinária, permitindo o maior detalhamento espacial do atributo RP.

\section{Resultados e Discussão}

Para regionalizar a dispersão dos pontos amostrados promoveu-se a análise do semivariograma de RP perfil (Tabela 3). Observase que o modelo esférico foi o que melhor se ajustou para os sistemas de preparo e velocidades de deslocamento; o que esta coerente com Cortez et al. (2014) quando analisaram RP em Argissolo Amarelo sob diferentes intensidades de tráfego de tratores. Marasca et al. (2011) e Aquino et al. (2014) ao estudarem variabilidade espacial de RP constataram maior ocorrência do modelo esférico para atributos físicos do solo. Os ajustes apresentaram altas porcentagens de significância que pode ser observado por meio 
dos coeficientes de determinação $\left(R^{2}\right)$ variando de 0,85 a 0,99 .

O efeito pepita (Co) variou de 0,001 a 0,290 (Tabela 3). Como os valores do efeito pepita estão próximos a zero, aumenta-se a exatidão de estimativas por meio da krigagem estando de acordo com Vieira (2000). Esse autor explica que quanto menor a variação ao acaso, mais exata é a estimativa. O GT+AA foi o sistema que apresentou maiores valores de Co, variando de 0,090 a 0,290 , enquanto que a GL22 apresentou uma constância nos valores $(0,010)$.

Nos alcances (a) obtidos verifica-se uma variação entre 0,38-0,62 m, o que permite afirmar que a malha amostral poderia ser de no mínimo 0,38 m de distância horizontal, uma vez que foi utilizada a de distância de $0,15 \mathrm{~m}$; ou seja, para a obtenção da mesma precisão dos dados amostrados, as malhas estudadas poderiam ter sido de no mínimo de $0,38 \mathrm{~m}$ e máximo de $0,62 \mathrm{~m}$ de distância entre os pontos horizontais amostrados ao longo da seção transversal de trabalho dos equipamentos agrícolas utilizados. Conforme Campos et at. (2013) como os valores de alcance (a) foram superiores ao estabelecido na malha original $(0,15 \mathrm{~m})$, estes valores encontrados podem torna-se referência em futuros estudos de atributos físicos.

Tabela 3. Dados do semivariograma para a resistência do solo à penetração nas camadas de solo avaliadas para os sistemas de preparo em função das velocidades de deslocamento

\begin{tabular}{|c|c|c|c|c|c|c|c|c|}
\hline $\begin{array}{l}\text { Velocidades } \\
\left(\mathrm{km} \mathrm{h}^{-1}\right)\end{array}$ & Modelo & Co & $\mathrm{Co}+\mathrm{C}$ & $a(m)$ & $\mathrm{R}^{2}$ & $S Q R$ & ADE & Classe \\
\hline \multicolumn{9}{|c|}{ Preparo } \\
\hline \multicolumn{9}{|c|}{ GT + ESC } \\
\hline 2,45 & Esférico & 0,001 & 2,69 & 0,56 & 0,85 & 1,130 & 1,00 & Forte \\
\hline 3,88 & Esférico & 0,184 & 1,60 & 0,54 & 0,93 & 0,110 & 0,88 & Forte \\
\hline 5,72 & Esférico & 0,060 & 2,09 & 0,51 & 0,96 & 0,090 & 0,97 & Forte \\
\hline 6,50 & Esférico & 0,060 & 3,52 & 0,53 & 0,99 & 0,050 & 0,98 & Forte \\
\hline \multicolumn{9}{|c|}{$\mathrm{GT}+\mathrm{AA}$} \\
\hline 2,45 & Esférico & 0,130 & 1,06 & 0,38 & 0,88 & 0,047 & 0,87 & Forte \\
\hline 3,88 & Esférico & 0,090 & 2,02 & 0,55 & 0,99 & 0,013 & 0,95 & Forte \\
\hline 5,72 & Esférico & 0,260 & 2,45 & 0,53 & 0,99 & 0,025 & 0,89 & Forte \\
\hline 6,50 & Esférico & 0,290 & 3,06 & 0,56 & 0,98 & 0,077 & 0,90 & Forte \\
\hline \multicolumn{9}{|c|}{ GL22 } \\
\hline 2,45 & Esférico & 0,010 & 3,26 & 0,61 & 0,98 & 0,158 & 0,99 & Forte \\
\hline 3,88 & Esférico & 0,010 & 4,99 & 0,62 & 0,97 & 0,552 & 0,99 & Forte \\
\hline 5,72 & Esférico & 0,010 & 4,78 & 0,60 & 0,97 & 0,557 & 0,99 & Forte \\
\hline 6,50 & Esférico & 0,010 & 4,41 & 0,60 & 0,97 & 0,460 & 0,99 & Forte \\
\hline \multicolumn{9}{|c|}{ GL24 } \\
\hline 2,45 & Esférico & 0,010 & 3,82 & 0,55 & 0,96 & 0,451 & 0,99 & Forte \\
\hline 3,88 & Esférico & 0,010 & 4,10 & 0,52 & 0,98 & 0,185 & 0,99 & Forte \\
\hline 5,72 & Esférico & 0,010 & 4,17 & 0,52 & 0,95 & 0,707 & 0,99 & Forte \\
\hline 6,50 & Esférico & 0,039 & 2,65 & 0,53 & 0,98 & 0,089 & 0,98 & Forte \\
\hline \multicolumn{9}{|c|}{ SPP } \\
\hline & Esférico & 0,240 & 2,42 & 0,49 & 0,98 & 0,036 & 0,90 & Forte \\
\hline
\end{tabular}

$\mathrm{Co}$ = efeito pepita; $\mathrm{Co}+\mathrm{C}$ = patamar; $\mathrm{a}=$ alcance; $\mathrm{R}^{2}$ = coeficiente de determinação; $\mathrm{SQR}=$ soma dos quadrados dos desvios; $\mathrm{ADE}=$ avaliador da dependência espacial. GT+ESC: grade tandem + escarificador; GT+AA: grade tandem + arado de aivecas; GL22: grade off-set - discos de 0,56 m; GL24: grade off-set - discos de 0,61 m; SPP: sem GT+ESC: grade tande
preparo primário.

- grau de dependência espacial (ADE) pode ser classificado conforme Zimback (2001), em forte, por estarem acima de 0,75 e uma vez que, os valores variam de 0,87 a 1,00. Segundo Neves Neto et al. (2013) quanto maior a dependência espacial do atributo, melhor estrutura espacial e maior precisão podem ser obtidas na estimativa em pontos não amostrados. Diferentemente de Silva et al.
(2009) que ao estudar variabilidade espacial em Latossolo Vermelho concluíram que o preparo convencional e plantio direto não influenciaram a dependência espacial da RP.

Na avaliação dos erros de estimativa promoveu-se a validação cruzada dos dados de RP (Tabela 4), permitindo a comparação de valores, estimados e amostrados, por meio das informações disponíveis. 
Tabela 4. Dados da validação cruzada para a resistência do solo à penetração nas camadas de solo avaliadas para os sistemas de preparo em função das velocidades de deslocamento

\begin{tabular}{|c|c|c|c|}
\hline \multirow{2}{*}{ Velocidades $\left(\mathrm{km} \mathrm{h}^{-1}\right)$} & \multicolumn{3}{|c|}{ Validação cruzada } \\
\hline & $\mathrm{R}^{2}$ & $A$ & $b$ \\
\hline \multicolumn{4}{|c|}{ Preparo } \\
\hline \multicolumn{4}{|c|}{$\mathrm{GT}+\mathrm{ESC}$} \\
\hline 2,45 & 0,97 & $-0,001$ & 1,00 \\
\hline 3,88 & 0,90 & $-0,027$ & 1,02 \\
\hline 5,72 & 0,88 & 0,017 & 0,98 \\
\hline 6,50 & 0,96 & $-0,013$ & 1,01 \\
\hline \multicolumn{4}{|c|}{$\mathrm{GT}+\mathrm{AA}$} \\
\hline 2,45 & 0,86 & $-0,025$ & 1,03 \\
\hline 3,88 & 0,97 & $-0,017$ & 1,02 \\
\hline 5,72 & 0,89 & $-0,033$ & 1,03 \\
\hline 6,50 & 0,94 & $-0,031$ & 1,03 \\
\hline \multicolumn{4}{|c|}{ GL22 } \\
\hline 2,45 & 0,96 & 0,016 & 0,99 \\
\hline 3,88 & 0,97 & $-0,004$ & 1,00 \\
\hline 5,72 & 0,96 & $-0,010$ & 1,00 \\
\hline 6,50 & 0,98 & $-0,003$ & 1,00 \\
\hline \multicolumn{4}{|c|}{ GL24 } \\
\hline 2,45 & 0,97 & $-0,001$ & 1,00 \\
\hline 3,88 & 0,97 & $-0,004$ & 1,00 \\
\hline 5,72 & 0,96 & $-0,010$ & 1,00 \\
\hline 6,50 & 0,98 & $-0,003$ & 1,00 \\
\hline \multicolumn{4}{|c|}{ SPP } \\
\hline & 0,91 & $-0,027$ & 1,01 \\
\hline
\end{tabular}

Verifica-se que os coeficientes angulares na validação cruzada para os sistemas de preparo do solo e para as quatro velocidades de deslocamento (Tabela 4) apresentam-se próximos ou iguais a 1,0. Isso indica proporcionalidade entre os valores amostrados e estimados conforme afirmado por Carvalho et al. (2006). Pode-se observar que o coeficiente de determinação $\left(R^{2}\right)$ foi elevado para as velocidades de deslocamento, que apresentaram valor igual a 1,0 para o coeficiente angular, e a intersecção (A) mais próxima de zero.

Nos mapas de isolinhas (Figuras 1, 2, 3, 4 e 5) com os valores de RP agrupados em 05 classes de cores de ordem crescente podem-se verificar os padrões de RP no perfil do solo após o manejo com os equipamentos e velocidades de deslocamento.

A partir dos mapas de isolinhas podese predizer qual o sistema de preparo do solo mais eficiente para a mobilização do solo sem que haja ocorrência de formação de camada compactada e/ou depauperamento dos atributos do solo; dentre os sistemas utilizados destacam-se o GT+ESC e GT+AA. Concordando com Coelho et al. (2012) e Rodrigues et al. (2014) ao afirmarem que o zoneamento da compactação (RP) em regiões específicas possibilita o manejo adequado do solo.

Por meio dos mapas, também se pode verificar que a RP aumenta, à medida que a profundidade aumenta. Situação semelhante foi verificada por Carvalho et al. (2008) ao avaliarem a variabilidade espacial da RP e do conteúdo de água em Latossolo Vermelho argiloso sob sistemas de manejo de colheita mecanizada de cana-de-açúcar; e por Coelho et al. (2012) ao avaliar a variabilidade espacial da RP em área cultivada com manga em Vertissolo na região de Juazeiro-BA, e também por Rodrigues et al. (2014) ao avaliar o padrão de distribuição espacial da resistência do solo à penetração em área de capineira irrigada em Argissolo Amarelo.

O aumento da RP em profundidade, provavelmente, ocorreu devido à acomodação natural das argilas do solo, e a gradual diminuição da matéria orgânica e da atividade 

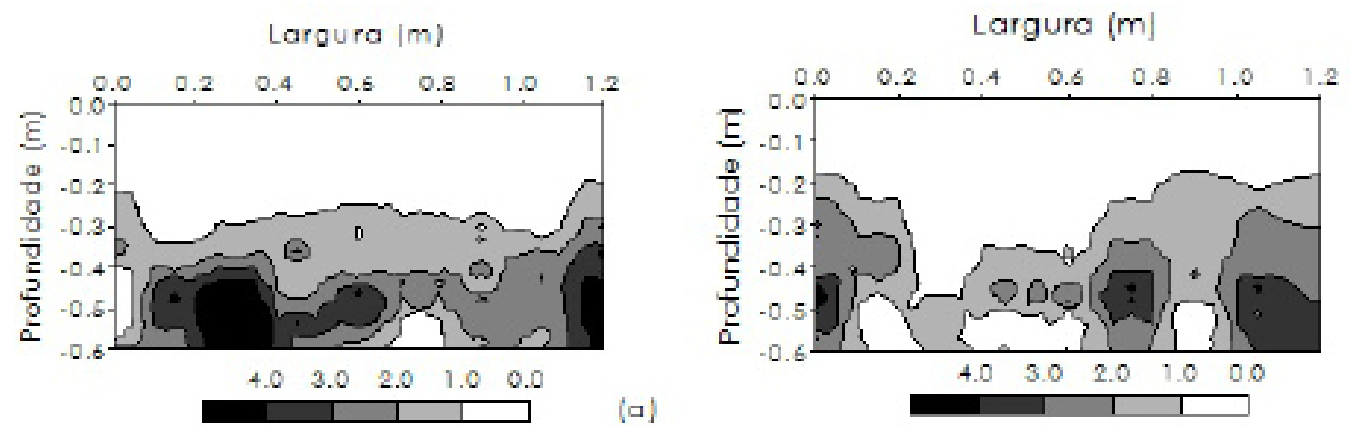

(b)
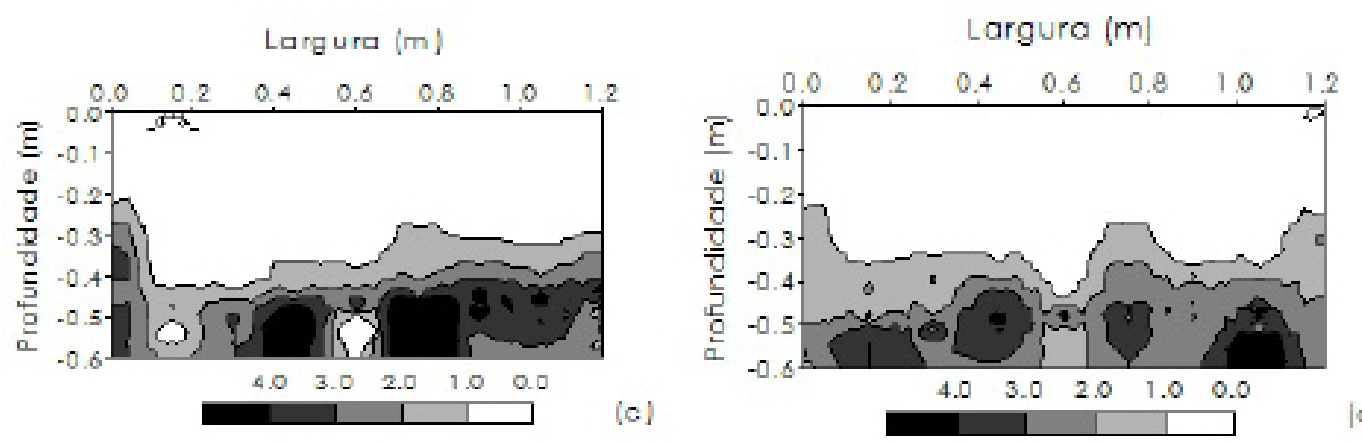

Figura 1. Mapas de isolinhas do escarificador (GT + ESC) para RP perfil (MPa) nas velocidades de deslocamento: (a) 2,45 $\mathrm{km} \mathrm{h}^{-1}$ (b) $3,88 \mathrm{~km} \mathrm{~h}^{-1}$ (c) $5,72 \mathrm{~km} \mathrm{~h}^{-1} \mathrm{e}$ (d) $6,50 \mathrm{~km} \mathrm{~h}^{-1}$.
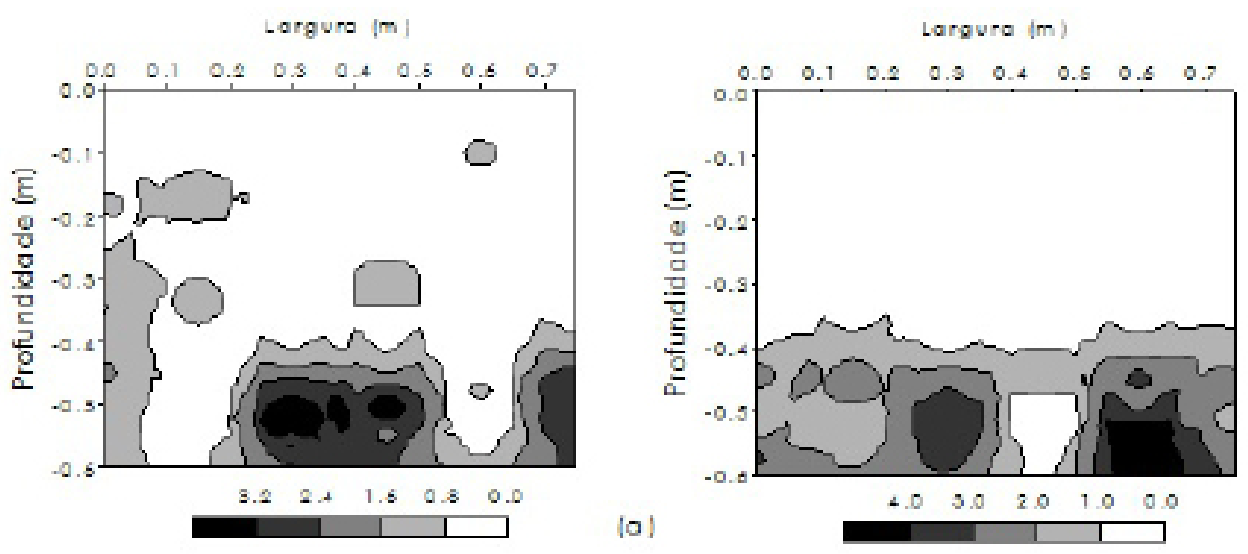

(b)
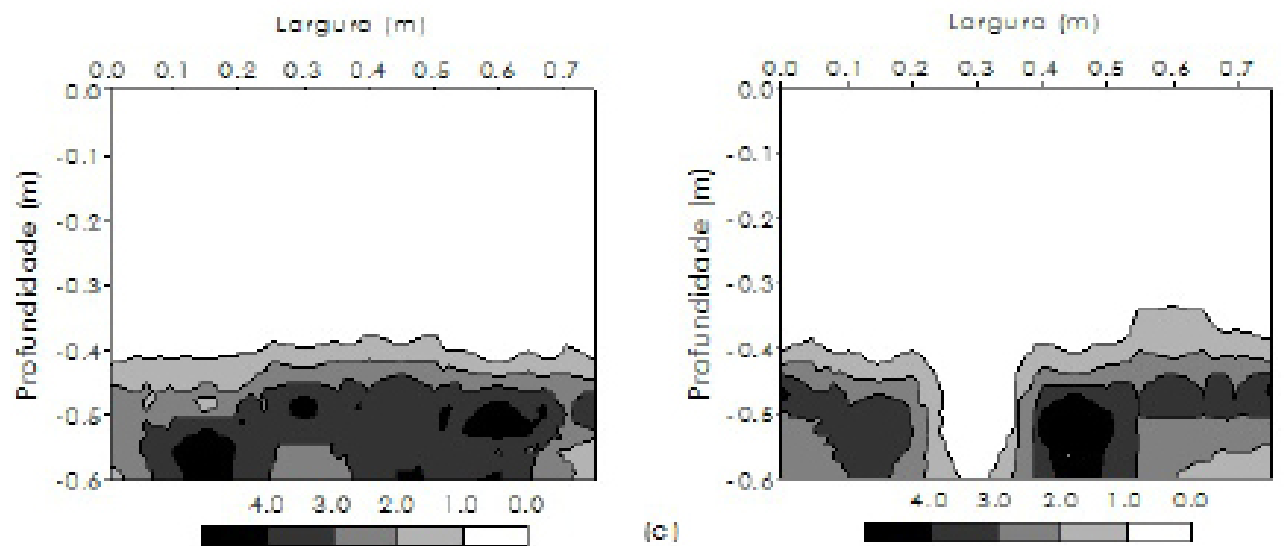

(d)

Figura 2. Mapas de isolinhas do arado de aivecas (GT + AA) para RP perfil (MPa) nas velocidades de deslocamento: (a) $2,45 \mathrm{~km} \mathrm{~h}^{-1}$; (b) $3,88 \mathrm{~km} \mathrm{~h}^{-1}$; (c) $5,72 \mathrm{~km} \mathrm{~h}^{-1} \mathrm{e}$ (d) $6,50 \mathrm{~km} \mathrm{~h}^{-1}$. 

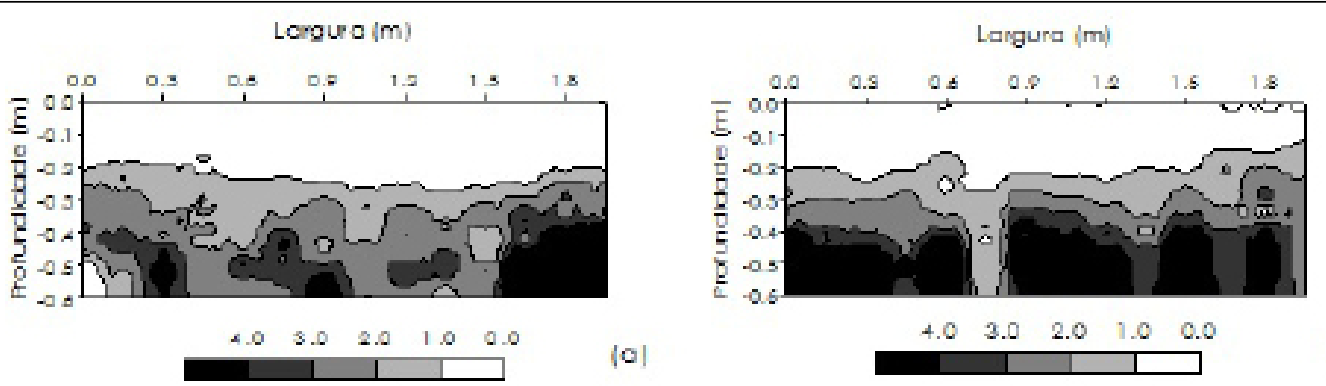

(b)
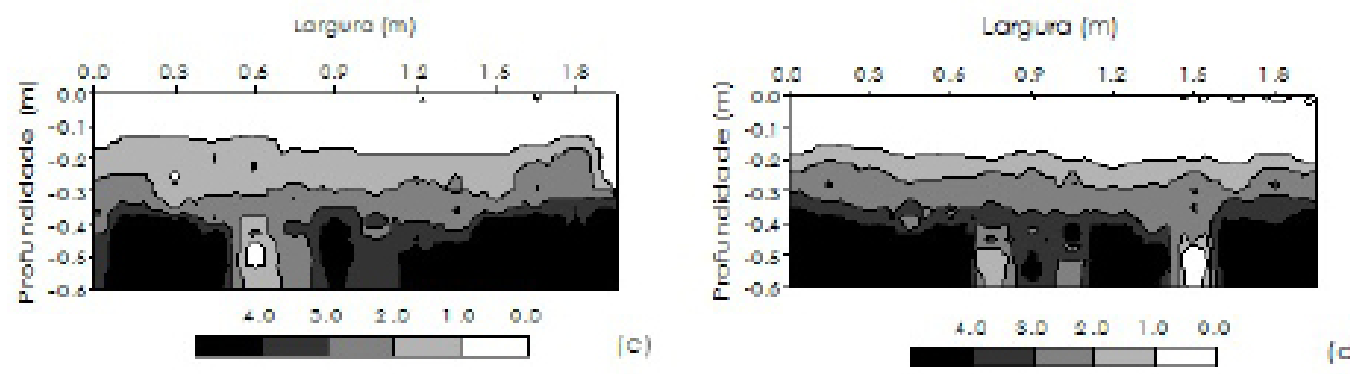

(d)

Figura 3. Mapas de isolinhas da grade off-set de discos de 0,56 $\mathrm{m}$ (GL22) para RP perfil (MPa) nas velocidades de deslocamento: (a) $2,45 \mathrm{~km} \mathrm{~h}^{-1}$; (b) $3,88 \mathrm{~km} \mathrm{~h}^{-1}$; (c) $5,72 \mathrm{~km} \mathrm{~h}^{-1}$ e (d) $6,50 \mathrm{~km} \mathrm{~h}^{-1}$.
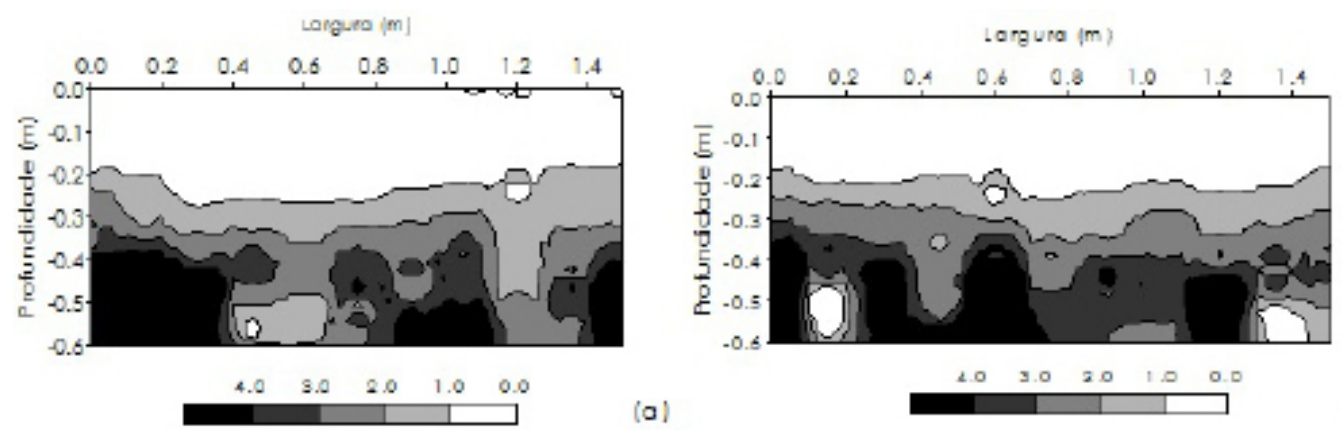

(b)
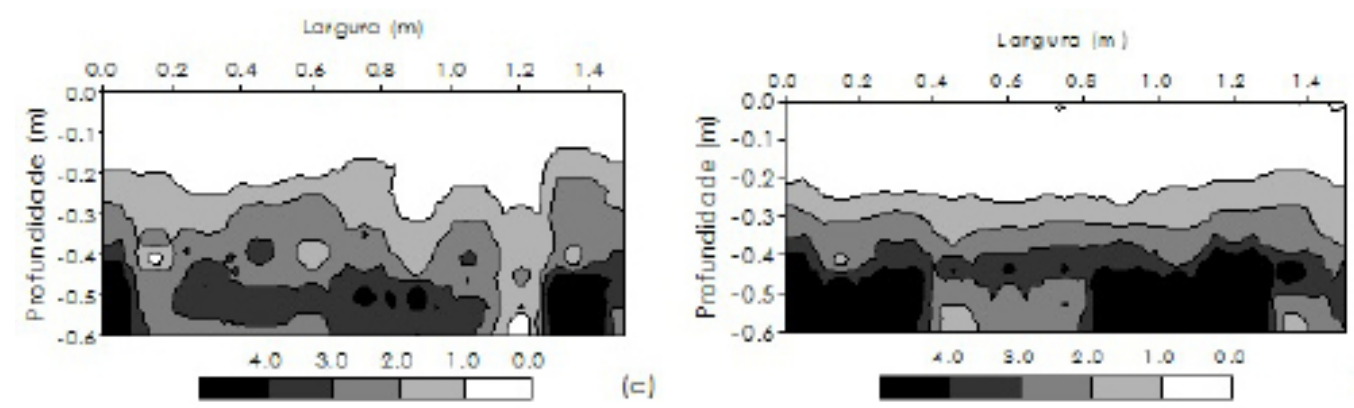

Figura 4. Mapas de isolinhas da grade off-set de discos de 0,61 $\mathrm{m}$ (GL24) para RP perfil (MPa) nas velocidades de deslocamento: (a) $2,45 \mathrm{~km} \mathrm{~h}^{-1}$; (b) $3,88 \mathrm{~km} \mathrm{~h}^{-1}$; (c) $5,72 \mathrm{~km} \mathrm{~h}^{-1}$ e (d) $6,50 \mathrm{~km} \mathrm{~h}^{-1}$.

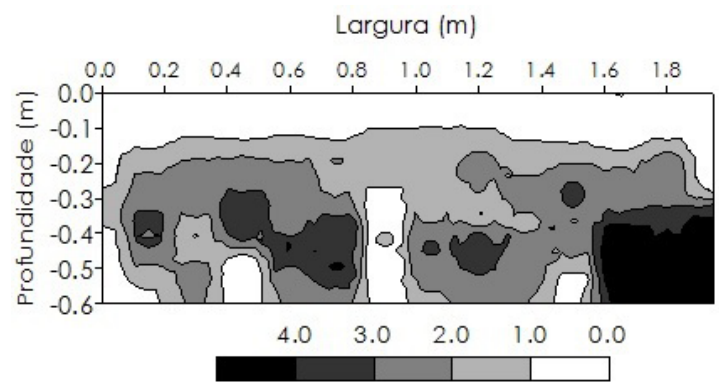

Figura 5. Mapa de isolinhas do sistema sem preparo primário (SPP) para RP perfil (MPa). 
microbiológica ao longo do perfil do solo. Neves Neto et al. (2013) ao analisarem atributos do solo e cobertura vegetal em diferentes condições de pastagem verificaram que a RP nas áreas de pastagens com alto grau de degradação apresentaram-se elevadas quando comparadas com a área de vegetação nativa. Ao contrário Carvalho et al. (2008) atribuíram provável causa da compactação, à pressão exercida no solo em conseqüência do sistema de colheita mecanizado da cana.

Além, disso, os equipamentos com maior profundidade efetiva (escarificador e arado de aivecas) foram os que promoveram rompimento dessas zonas compactadas. Enquanto que os equipamentos de maior largura efetiva (grades off-set GL22 e GL24) propiciaram maior uniformização superficial da área mobilizada.

Para as grades off-set tem-se os maiores valores de RP a partir da camada 0,30 m em seus mapas de isolinhas, que segundo Nagahama et al. (2013) já era esperado visto que estas possuem órgãos ativos de menor penetração. Entretanto, não se observou conforme Sene et al. (1985), valor limite para desenvolvimento radicular de 6,0 MPa, ou seja, para as grades offset considerando os valores encontrados podese verificar que estes estão abaixo deste valor limite.

Por meio dos mapas de isolinhas, é possível observar os equipamentos e suas respectivas profundidades de trabalho, sendo que $o$ escarificador $e$ arado de aivecas trabalharam abaixo de 0,30 e $0,40 \mathrm{~m}$, respectivamente. Enquanto, as grades off-set em torno de $0,20 \mathrm{~m}$, e o sem preparo primário que teve o preparo com grade tandem (preparo secundário) foi menor que $0,15 \mathrm{~m}$; com isto, atendem as regulagens pré-definidas. Silva et al. (2009) encontraram valores distintos de RP para dois sistemas de preparo do solo (plantio direto e preparo convencional) em Latossolo Vermelho distrófico e Cortez et al. (2011) concluíram que a RP em Argissolo Amarelo apresentou valores adequados até a profundidade de trabalho dos equipamentos.

Os valores de RP encontrados não são restritivos ao desenvolvimento radicular, por estarem abaixo de 6,0 MPa (Sene et al., 1985), com RP superficial não superior a 2,0 MPa; diferentemente de Almeida et al. (2008) que verificaram RP média de 0,47 MPa para umidade gravimétrica de $0,09 \mathrm{~kg} \mathrm{~kg}^{-1}$. Mas, todavia, devese estar atento ao manejo da irrigação, uma vez que o horizonte B textural do Argissolo Amarelo possui teor de argila maior que o horizonte $A \mathrm{e}$ com isto, podendo dificultar a infiltração da água resultando em saturação da camada superficial. Para áreas com maior declive, pode ocorrer aceleração do processo de perdas de solo por erosão, quando mobilizados, sendo seu uso mais adequado para o cultivo de culturas perenes.

\section{Conclusões}

O sistema grade tandem mais arado de aivecas foi o sistema que apresentou menores valores de resistência do solo à penetração nas camadas superficiais do solo.

A presença de zonas com maiores valores de resistência solo à penetração concentrou-se em sistemas de preparo do solo com equipamentos de menor profundidade efetiva de trabalho (grades).

Por meio dos mapas de isolinhas podese verificar a área de atuação dos órgãos ativos dos equipamentos agrícolas utilizados.

A resistência mecânica do solo à penetração não mostrou restritiva ao desenvolvimento radicular para $\bigcirc$ Argissolo Amarelo por estar abaixo de 6,0 MPa.

\section{Agradecimentos}

Ao CNPq - Conselho Nacional de Desenvolvimento Científico e Tecnológico pelas bolsas de Iniciação Científica do terceiro e quarto autor. A FACEPE - Fundação de Amparo a Ciência e Tecnologia do Estado de Pernambuco pela Bolsa de Iniciação Científica do quinto autor. Ao CNPa pela concessão de Bolsa de Produtividade do segundo autor.

\section{Referências}

Almeida, C.X., Centurion, J.F., Freddi, O.S., Jorge, R.F., Barbosa, J.C. 2008. Funções de pedotransferência para a curva de resistência do solo à penetração. Revista Brasileira de Ciência do Solo 32: 2235-2243.

Alves, S.M.F., Queiroz, D.M., Alcântara, G.R., Reis, 
E.F. 2014. Variabilidade espacial de atributos físicoquímicos do solo usando técnicas de análise de componentes principais e geoestatística. Bioscience Journal 30:22-30.

Amaral, F.C.S., Silva, E.F., Melo, A.S. 2006. Caracterização pedológica e estudos de infiltração da água no solo em perímetros irrigados no Vale do São Francisco. Embrapa Solos, Rio de Janeiro, Brasil. 104p.

Aquino, R.E., Campos, M.C.C., Oliveira, I.A. Marques Júnior, J., Silva, D.M.P., Silva, D.A.P. 2014. Variabilidade espacial de atributos físicos de solos antropogênico e não antropogênico na região de Manicoré, AM. Bioscience Journal 30: 988-997.

Araújo, A.O., Mendonça, L.A.R., Lima, M.G.S., Feitosa, J.V., Silva, F.J.A., Ness, R.L.L., Frischkorn, H., Simplício, A.A.F, Kerntopf, M.R. 2013. Modificações nas propriedades dos solos de uma área de manejo florestal na Chapada do Araripe. Revista Brasileira de Ciência do Solo 37: 754-762.

Asae. American Society Of Agricultural Engineers. 2004. Soil cone penetrometer - ASAE S 313.3. ASAE, St. Joseph, USA. 2 p.

Campos, M.C.C., Soares, M.D.R., Santos, L.A.C., Oliveira, I.A., Aquino, R.E., Bergamin, A.C. 2013. Variabilidade espacial dos atributos físicos em um Argissolo Vermelho sob floresta. Comunicata Scientiae 4: 168-178.

Carvalho, G.J., Carvalho, M.P., Freddi, O.S., Martins, M.V. 2006. Correlação da produtividade do feijão com a resistência à penetração do solo sob plantio direto. Revista Brasileira de Engenharia Agrícola e Ambiental 10: 765-771.

Carvalho, L.A., Neto, V.J.M., Silva, C.F., Pereira, J.G., Nunes, W.A.G.A., Chaves, C.H.C. 2008. Resistência mecânica do solo à penetração (RMP) sob cultivo de cana-de-açúcar, no município de Rio Brilhante - MS. Agrarian 1: 7-22.

Coelho, D.S., Cortez, J.W., Olszevski, N. 2012. Variabilidade espacial da resistência mecânica à penetração em Vertissolo cultivado com manga no perímetro irrigado de Mandacaru, Juazeiro, Bahia, Brasil. Revista Brasileira de Ciência do Solo 36: 755-763.

Cortez, J.W., Alves, A.D.S., Moura, R.D., Olszevski, N., Nagahama, H.J. 2011. Atributos físicos do Argissolo amarelo do semiárido nordestino sob sistemas de preparo. Revista Brasileira de Ciência do Solo 35: 1207-1216.

Cortez, J.W., Olszevski, N., Pimenta, W.A., Patrocínio Filho, A.P., Souza, E.B., Nagahama, H.J. 2014. Avaliação da intensidade de tráfego de tratores em alguns atributos físicos de um Argissolo Amarelo. Revista Brasileira de Ciência do Solo 38: 1000-1010.

Embrapa. Empresa Brasileira de Pesquisa Agropecuária. 2013. Sistema Brasileiro de Classificação de Solos. Centro Nacional de Pesquisas de Solos, Brasília, Brasil. 353p.

Falker. 2010. PenetroLOG PLG1020 - Medidor Eletrônico de Compactação do Solo. Disponível em: < http://www.falker.com.br/download.php> . Acesso em: 23 set.

Magalhães, W.A, Cremon, C., Mapeli, N.C., Silva, W.m., Carvalho, J.M., Mota, M.S. 2009. Determinação da resistência do solo a penetração sob diferentes sistemas de cultivo em um Latossolo sob Bioma Pantanal. Agrarian 2: 21-32.

Marasca, I., Oliveira, C.A.A., Guimarães, E.C., Cunha, J.P.A.R., Assis, R.L., Perin, A., Menezes, L.A.S. 2011 . Variabilidade espacial da resistência do solo à penetração e teor de agua em sistema de plantio direto na cultura da soja. Bioscience Journal 27: 239-246.

Mion, R.L., Nascimento, E.M.S., Sales, F.A.L., Silva, S.F., Duarte, J.M.L., Sousa, B.M. 2012. Variabilidade espacial da porosidade total, umidade e resistência do solo à penetração de um Argissolo Amarelo. Semina. Ciências Agrárias 33: $2057-$ 2066.

Modolo, A.J., Fernandes, H.C., Schaefer, G.C.E., Silveira, J.C.M. 2008. Efeito da compactação do solo sobre a emergência de plântulas de soja em sistema semeadura direta. Ciência e Agrotecnologia 32: 1259-1265.

Nagahama, H.J., Cortez, J.W., Pimenta, W.A., Patrocínio Filho, A.P., Souza, E.B. 2013. Desempenho do conjunto trator-equipamento em sistemas de preparo periódico no Argissolo Amarelo. Revista Energia na Agricultura 28: 79-89.

Neves Neto, D.N., Santos, A.C., Santos, P.M., Melo, J.C., Santos, J.S. 2013. Análise espacial de atributos do solo e cobertura vegetal em diferentes condições de pastagem. Revista Brasileira de Engenharia Agrícola e Ambiental 17: 995-1004.

Oliveira, I.A., Campos, M.C.C., Soares, M.P.R., Aquino, R.E., Marques Júnior, J., Nascimento, E.P. 2013. Variabilidade espacial de atributos físicos em um Cambissolo Háplico sob diferentes usos na região sul do Amazonas. Revista Brasileira de Ciência do Solo 37: 1103-1112.

Rodrigues, M.S., Ramos, R.R.D., Azevedo, T.P., Patrocínio Filho, A.P., Oliveira, L.G. 2014. Variabilidade espacial da resistência do solo à penetração em área de capineira irrigada no semiárido. Agropecuária Científica no Semiárido 10: 161-166. 
Sene, M., Vepraskas, M.J., Naderman, G.C., Denton, H.P. 1985. Relationship of soil texture and structure to corn yield response to subsoiling. Soil Science Society of America Journal 49: 422-427.

Silva, J.M., Lima, J.S.S., Pires, F.R., Assis, R.L. 2008. Variabilidade espacial dos atributos físicos em um Latossolo sob plantio direto e preparo convencional no cultivo da soja no cerrado. Revista de Ciências Agrárias 50: 167-180.

Silva, J.M., Passos, A.L.R., Beltrão, F.A.S. 2009. Análise espacial da densidade, umidade e resistência mecânica do solo à penetração sob sistemas de cultivo. Engenharia Ambiental 6: 103118.

Stefanoski, D.C., Santos, G.C., Marchão, R.L., Petter, F.A., Pacheco, L.P. 2013. Uso e manejo do solo e seus impactos sobre a qualidade física. Revista Brasileira de Engenharia Agrícola e Ambiental 17: 1301-1309.

Stolf, R. 1991. Teoria e teste experimental de formulas de transformação dos dados de penetrômetro de impacto em resistência do solo. Revista Brasileira de Ciência do Solo 15: 229235.

Stone, A.J., Guimarães, C.M., Moreira, J.A.A. 2002. Compactação do solo na cultura do feijoeiro - 1: efeitos nas propriedades físico-hídricas do solo. Revista Brasileira de Engenharia Agrícola e Ambiental 6: 207-212.

Vieira, S.R. 2000. Geoestatística em estudos de variabilidade espacial do solo. In: Novais, R.F., Alvarez, V.H., Schaefer, C.E.G.R. (ed.) Tópicos em Ciência do Solo. Sociedade Brasileira de Ciência do Solo, Viçosa, Brasil. p.1-53 (volume 1).

Zimback, C.R.L. 2001. Análise espacial de atributos químicos de solos para fins de mapeamento da fertilidade do solo. 114f. Tese (Doutorado) Universidade Estadual Paulista, Botucatu, Brasil. 